\title{
Inhaltsverzeichniss des neunundsiebzigsten Bandes.
}

Ueber die constante elektriscbe Strömung in ebenen Platten. Von Herrn

E. Heine in Halle. . . . . . . . . . . . . . . . . . . . Seite 1

Extrait d'une lettre de M. Ch. Hermite à M. Borchardt sur la réduction des formes quadratiques ternaires. . . . . . . . . . . . . . . . -17

Versuch einer Classification der willkurlichen Functionen rceller Argumente nach ibren Aenderungen in den kleinsten Intervallen. Von Herrn Paul du Bois-Reymond in Ttibingen. . . . . . . . . . . . . - 21

Allgemeine Lebrsätze tuber den Gultigkeitsbereich der Integralformeln, die zur Darstellung willkürlicher Functionen dienen. Von Demselben. . - 38

Zur Theorie der Eulerschen Zahlen. Von Herrn Stern in Göttingen. . . - 67

Erzeugnisse, Elementarsysteme und Charakteristiken von cubischen Raumcurven. Von Herrn Rudolf Sturm in Darmstadt. . . . . . . . . . - 99

Ueber eine reciproke Verwandtschaft des zweiten Grades. Von Herrn Milinowski in Weissenburg im Elsass. . . . . . . . . . . . . -140

Ueber algebraische Flächen, die zu einander apolar sind. Von Herrn Th. Reye in Strassburg i./E. . . . . . . . . . . . . . . . . . . -159

New Demonstration of the Reduction of Hyperelliptic Integrals to the Normal

Form. By John C. Malet. Trinity-College Dublin. . . . . . . . - 176

Ueber die Multiplicationsregel für zwei unendliche Reihen. Von Herrn F. Mertens in Krakau. . . . . . . . . . . . . . . . . . -182

Anwendungen der Determinantentheorie auf die Geometrie des Maasses. Von Herrn G. Frobenius. . . . . . . . . . . . . . . . . . . . - 185

Sur la limite du degré des groupes primitifs qui contiennent une substitution donnée. Par M. Camille Jordan à Paris. . . . . . . . . . . . -248

Ueber eine veränderte Form der Bedingung futr die Integrirbarkeit der Functionen. Von Herrn Paul du Bois-Reymond in Tubingen. . . . - 259

Ueber den Werth einiger Integrale. Von Herrn Stern in Göttingen. . . . - 263 
Zur Theorie der Potentialflächen unter besonderer Rücksicht auf Körper, die von Flächen der zweiten Ordnung begrenzt sind. Von Herrn $W$. Stahl in Aachen. . . . . . . . . . . . . . . . . . . . . . . . . Seite 265 Ueber Curven, deren Bogen ein elliptisches Integral erster Gattung ist. Von Herrn L. Kiepert in Freiburg i. Br. . . . . . . . . . . . . . - 304 Extrait d'une lettre de M. Ch. Hermite de Paris à M. L. Fuchs de Gottingue sur quelques équations différentielles linéaires. . . . . . . . . . -324

Lettre de M. Ch. Hermite à M. Borchardt sur la fonction de Jacob Bernouilli. - 339 Otto Hesse. Von C. W. Borchardt. . . . . . . . . . . . . . . . -345 